\title{
POWER-BOUNDED ELEMENTS AND RADICAL BANACH ALGEBRAS
}

\author{
GRAHAM R. ALLAN \\ Department of Pure Mathematics and Mathematical Statistics \\ University of Cambridge \\ 16 Mill Lane, Cambridge, CB2 1SB, England \\ E-mail: gra3@pmms.cam.ac.uk
}

\begin{abstract}
Firstly, we give extensions of results of Gelfand, Esterle and Katznelson-Tzafriri on power-bounded operators. Secondly, some results and questions relating to power-bounded elements in the unitization of a commutative radical Banach algebra are discussed.
\end{abstract}

1. Introduction. In what follows, $A$ is a complex Banach algebra with an identity element, $\Delta$ is the closed unit disc in the complex plane, and $\Gamma$ is the boundary of $\Delta$. For any element $x$ of $A$, let $\operatorname{Sp} x$ be the spectrum of $x$ in $A$ and let $r(x)$ be the spectral radius of $x$. We say that an element $x$ of $A$ is power-bounded if and only if $\sup _{n \geq 1}\left\|x^{n}\right\|<\infty$. If $x$ is an invertible element of $A$, then $x$ is called doubly power-bounded if and only if both $x$ and $x^{-1}$ are power-bounded. The well-known spectral radius formula implies that, if $x$ is power-bounded, then $r(x) \leq 1$; also, if $x$ is doubly power-bounded, then $\operatorname{Sp} x \subseteq \Gamma$. A further simple consequence of the formula is that $x^{n} \rightarrow 0$ as $n \rightarrow \infty$ if and only if $r(x)<1$. In 1986, Katznelson and Tzafriri [8] proved the following generalization of this elementary fact. (In fact in [8], the result is in an equivalent formulation in the language of operators. For our purposes, it is more natural to set results in the context of Banach algebras.)

Theorem 1 (Katznelson and Tzafriri). Let the element $x \in A$ be power-bounded. Then $x^{n}(x-1) \rightarrow 0$ as $n \rightarrow \infty$ if and only if $\operatorname{Sp} x \subseteq \operatorname{int} \Delta \cup\{1\}$.

Remark. The "only if" implication is trivial, and the other implication is trivial in case $1 \notin \operatorname{Sp} x$, since we would then have that $r(x)<1$.

In fact the non-trivial implication had already been proved by Esterle (1981) [4] for the case in which $\operatorname{Sp} x=\{1\}$.

1991 Mathematics Subject Classification: Primary 47A10, 47A35, 47D03; Secondary 30B30, $30 \mathrm{D} 15$.

The paper is in final form and no version of it will be published elsewhere. 
Theorem 2 (Esterle). Let the element $x \in A$ be power-bounded with $\operatorname{Sp} x=\{1\}$. Then $x^{n}(x-1) \rightarrow 0$ as $n \rightarrow \infty$.

However, very much earlier, there was a partly analogous result of Gelfand (1941) [5].

Theorem 3 (Gelfand). Let $x \in A$ have $\operatorname{Sp} x=\{1\}$ and suppose that $x$ is doubly power-bounded. Then $x=1$.

Remark. This result of Gelfand is omitted from several well-known texts on the theory of Banach algebras. (It appears in Bourbaki's Théories Spectrales as an exercise!) The result is in the monograph of Hille-Phillips [7]. In fact Hille has the following strengthening of the result:

Theorem 3a (Hille). Let $x \in A$ have $\operatorname{Sp} x=\{1\}$ and suppose that $\left\|x^{n}\right\|=o(|n|)$ as $n \rightarrow \pm \infty$. Then $x=1$.

In view of Hille's result, it is natural to ask whether Theorems 1 and 2 can be similarly strengthened. It is, perhaps, of interest to note first the very simple fact that the condition $\left\|x^{n}\right\|=o(n)$ is a necessary condition for $x^{n}(x-1) \rightarrow 0$. For, in the latter case, we must also have

$$
\frac{1}{n}\left(x^{n+1}-x\right)=\frac{1}{n} \sum_{k=1}^{n} x^{k}(x-1) \rightarrow 0 \quad \text { as } n \rightarrow \infty,
$$

so that $\left\|x^{n}\right\|=o(n)$. However, in ([2], Theorem 4.2), an example is given in which $\operatorname{Sp} x \subseteq$ int $\Delta \cup\{1\},\left\|x^{n}\right\|=o(n)$, but $x^{n}(x-1) \not \rightarrow 0$, showing that such an extension of Theorem 1 is not possible. It is understood that Atzmon has an example (not yet seen by the author) in which $\operatorname{Sp} x=\{1\}$; this would show that there was no comparable extension of Theorem 2, either.

In [8], Theorem 1 (and various generalizations) were proved by methods of elementary harmonic analysis. Other proofs have been given: for example, in 1987, Allan, O'Farrell and Ransford [1] used complex-variable methods as a substitute for the harmonic analysis. In 1989, Allan and Ransford [2] showed that Theorem 1 (and its generalizations) may be deduced directly from Theorem 3; their method was greatly influenced by Esterle's proof of Theorem 2. There are also various generalizations in which one considers $\limsup _{n \rightarrow \infty}\left\|f(x) x^{n}\right\|$, for suitable functions $f$, and in which it is not required that $\operatorname{Sp} x \cap \Gamma=\{1\}$. Even when $f$ does not vanish on $\operatorname{Sp} x \cap \Gamma$, it is possible to give estimates for $\lim \sup _{n \rightarrow \infty}\left\|f(x) x^{n}\right\|$ (see e.g. [2]).

In the next section, we shall discuss some different types of extension.

2. Algebras with a seminorm. We shall give generalizations of Theorems 1 and 3 in which the boundedness assumptions (and the conclusions) are in terms of an auxiliary linear-space seminorm on the algebra. Of course, the extension to Theorem 1 includes a similar extension to Theorem 2 as a special case.

Firstly, we recall some complex analysis. Let $X$ be a complex Banach space and let $f: \mathbb{C} \rightarrow X$ be an $X$-valued entire function. We say that $f$ is of exponential type if and only if there are constants $C, A>0$ such that $\|f(z)\| \leq C e^{A|z|}$ for all complex $z$. The type of $f$ is the infimum of the constants $A$ for which such an equality holds (but where 
the constant $C$ may depend on $A$ ). In particular, we say that $f$ has zero (or minimal) exponential type if and only if, for every $\varepsilon>0$ there is a constant $C>0$ such that $\|f(z)\| \leq C e^{\varepsilon|z|}$ for all $z$. We need the following classical result:

LEMmA. Any entire function of minimal exponential type that is bounded on the integers is constant on $\mathbb{C}$.

Proof. See e.g. [3], Theorem (10.2.11).

Of course, the classical result is for complex-valued functions; the extension to vectorvalued functions is routine functional analysis.

Now let $A$ be a complex Banach algebra, let $b \in A$ and define $f(z)=e^{z b}$ for all $z$. Then it is very clear that $f$ is an entire $A$-valued function of exponential type, since obviously $\|f(z)\| \leq e^{\|b\||z|}$. However, it easily seen that in fact we have the stronger fact that the type of $f$ is precisely $r(b)$. In particular, if $r(b)=0$, then $f$ is a function of minimal exponential type.

First we give the extended form of Gelfand's result.

TheOREM 4. Let $A$ be a complex, unital Banach algebra and let $p$ be a continuous seminorm on $A$. Let $x \in A$ with $\operatorname{Sp} x=\{1\}$ and suppose that $\sup _{n \in \mathbb{Z}} p\left(x^{n}\right)<\infty$. Then $p(x-1)=0$.

P r o of. By elementary functional calculus, we can write $x=e^{b}$, for some $b \in A$ with $\operatorname{Sp} b=\{0\}$. Let $f(z)=e^{z b}(z \in \mathbb{C})$. By the above discussion, $f$ is an entire $A$-valued function of minimal exponential type. Now let $\varphi$ be any $p$-continuous linear functional on $A$ and set $F(z)=\varphi(f(z))(z \in \mathbb{C})$. Then $\varphi$ is also $\|\cdot\|$-continuous, so that $F$ is a complex-valued entire function of minimal exponential type.

If $n \in \mathbb{Z}$, then, for some constant $K$,

$$
|F(n)| \leq K p\left(e^{n b}\right)=K p\left(x^{n}\right) \leq K \sup _{n} p\left(x^{n}\right)<\infty .
$$

By the Lemma, $F$ is constant; in particular, $0=\varphi(f(1)-f(0))=\varphi(x-1)$, for every $p$ continuous linear functional $\varphi$. By the Hahn-Banach theorem, it follows that $p(x-1)=0$.

Application 1. Let $X$ be a Banach space, let $T \in L(X)$ be such that $\operatorname{Sp}(T)=\{1\}$. Suppose that, for some $x \in X$, the set $\left\{T^{n} x: n \in \mathbb{Z}\right\}$ is bounded. Then $T x=x$. This follows just by applying Theorem 4 , with $A=L(X)$ and $p(S)=\|S x\|$ for $S \in A$.

We shall now prove an analogous extension to the Katznelson-Tzafriri result. We need the following tauberian lemma from [8].

LEMMA. Let $\left(a_{n}\right)$ be a bounded sequence of complex numbers and let $f(z)=\sum_{n \geq 0} a_{n} z^{n}$ $(|z|<1)$. Suppose that every point of $\Gamma \backslash\{1\}$ is a regular point for $f$. Then $a_{n+1}-a_{n} \rightarrow 0$ as $n \rightarrow \infty$.

In fact we need to apply the Lemma when $\left(a_{n}\right)$ is a sequence in a complex normed space. As in [8], this extension comes from very standard methods of functional analysis, together with the observation that the convergence asserted in the Lemma is uniform over certain normal families of functions. 
TheOrem 5. Let $A$ be a complex, unital Banach algebra and let $p$ be a continuous seminorm on $A$. Let $x \in A$ with $\operatorname{Sp} x \subset$ int $\Delta \cup\{1\}$ and suppose that $\sup _{n \geq 1} p\left(x^{n}\right)<\infty$. Then $p\left(x^{n+1}-x^{n}\right) \rightarrow 0$ as $n \rightarrow \infty$.

Proof. Let $X=A / p^{-1}(0)$ and let $\pi: A \rightarrow X$ be the quotient mapping. Then $p$ is constant on each coset of $p^{-1}(0)$ and we may therefore define the norm $\tilde{p}$ on $X$ by $\tilde{p}(\pi(a))=p(a)$, for all $a \in A$.

Now the function $R(z)=(1-z x)^{-1}$ is well-defined and analytic on an open neighbourhood of $\Delta \backslash\{1\}$. Moreover, since for $|z|$ sufficiently small we have $R(z)=\sum_{n \geq 0} x^{n} z^{n}$, with norm-convergence, the series, being the Taylor series of $R$ about 0 , must converge on the whole of int $\Delta$.

Hence, the function $f(z)=(\pi \circ R)(z)=\sum_{n \geq 0} \pi\left(x^{n}\right) z^{n}$ is an analytic $X$-valued function on int $\Delta$, and every point of $\Gamma \backslash\{1\}$ is a regular point for $f$. Since $\tilde{p}\left(\pi\left(x^{n}\right)\right)=$ $p\left(x^{n}\right)$, the Taylor coefficients of $f$ are bounded in the normed space $(X ; \tilde{p})$. It follows from the Lemma that $p\left(x^{n+1}-x^{n}\right)=\tilde{p}\left(\pi\left(x^{n+1}\right)-\pi\left(x^{n}\right)\right) \rightarrow 0$.

Application 2. By using the same seminorm as in Application 1, we can deduce the following: let $T \in L(X)$ with $\operatorname{Sp} T \subset$ int $\Delta \cup\{1\}$. Let $x \in X$ and suppose that $\left\{T^{n} x\right\}_{n \geq 1}$ is bounded. Then $T^{n}(I-T) x \rightarrow 0$ as $n \rightarrow \infty$.

Remark. Although it is the case that Theorem 3 may be very easily deduced from Theorem 1, and also (as in [2]) Theorem 1 may be deduced from Theorem 3, for the more general Theorems 4 and 5 , there does not seem to be any obvious way to deduce either from the other.

Theorem 6. Let $A$ be a complex, unital Banach algebra and let $x \in A$ with $\operatorname{Sp} x \subset$ int $\Delta \cup\{1\}$. Then, for all $\varepsilon>0$ there are integers $k \geq 0, N \geq 1$ and elements $a_{0}, a_{1}, \ldots, a_{N}$ of $A$ (which may all be taken to be polynomials in $x$ ) such that

(i) $\sum_{i=0}^{N}\left\|a_{i}\right\|<\varepsilon$,

(ii) $x^{k}(x-1)=\sum_{i=0}^{N} a_{i} x^{i}$.

Proof. Without loss of generality, we may take $A=A(x)$, the closed unital subalgebra of $A$ generated by $x$, since the given conditions on $\operatorname{Sp} x$ will apply also to the spectrum relative to this subalgebra.

Next, for all $y \in A$, define

$$
\left.p(y)=\inf \left\{\sum_{i=0}^{N}\left\|a_{i}\right\|: N \geq 1, a_{0}, \ldots, a_{N} \in A \text { such that } x^{k} y=\sum_{i=0}^{N} a_{i} x^{i} \text { (some } k\right)\right\} .
$$

Firstly, given $y \in A$, we may take $a_{0}=y$ and $a_{i}=0$ for $i \geq 1$. So $p$ is well-defined and $p(y) \leq\left\|a_{0}\right\|=\|y\|$. Also it is simple to see that $p$ is a (sub-multiplicative) seminorm on $A$ and that $p(x y)=p(y)$ for all $y \in A$. In particular, therefore, $p$ is a continuous seminorm on $A$ for which the sequence $\left(p\left(x^{k}\right)\right)$ is bounded (since $p\left(x^{k}\right)=p(1)$ for all $k \geq 1$ ). From Theorem 5 , we deduce that $p\left(x^{k}(x-1)\right) \rightarrow 0$ as $k \rightarrow \infty$.

Now let $\varepsilon>0$ and choose $k_{1}$ such that $p\left(x^{k_{1}}(x-1)\right)<\varepsilon$. By the definition of $p$ there are finitely many elements $a_{0}, \ldots, a_{N}$ of $A$ such that both $\sum_{i=0}^{N}\left\|a_{i}\right\|<\varepsilon$ and $x^{k}(x-1)=\sum_{i=0}^{N} a_{i} x^{i}$, for some $k \geq k_{1}$. 
To see that each of the $a_{i}$ may be taken as a polynomial in $x$, note that, since we initially reduced to the case where $A=A(x)$, we may, for each $i=0, \ldots, N$, choose a polynomial, say $q_{i}$ so that $\left\|a_{i}-q_{i}(x)\right\|$ is so small that both $\sum_{i=0}^{N}\left\|q_{i}(x)\right\|<\varepsilon$ and also $\left\|x^{k}(x-1)-\sum_{i=0}^{N} q_{i}(x) x^{i}\right\|<\varepsilon$. Then take $p_{0}(x)=q_{0}(x)+x^{k}(x-1)-\sum_{i=0}^{N} q_{i}(x) x^{i}$ and $p_{i}(x)=q_{i}(x)$ for $i \geq 1$ and we have $x^{k}(x-1)=\sum_{i=0}^{N} p_{i}(x) x^{i}$ and $\sum_{i=0}^{N}\left\|p_{i}(x)\right\|<2 \varepsilon$, which is obviously good enough.

As an application, we give a proof of a generalization of Theorem 1, proved by Allan and Ransford in [2].

Corollary. Let $A$ be a unital Banach algebra, let $x \in A$ with $\operatorname{Sp} x \subset \operatorname{int} \Delta \cup\{1\}$. Suppose that $\left\|x^{n}\right\| \leq \mu(n)$, for all $n \geq 0$, where $(\mu(n))$ is a sequence of positive numbers such that $\mu(n+1) / \mu(n) \rightarrow 1$ as $n \rightarrow \infty$. Then

$$
\mu(n)^{-1}\left\|x^{n+1}-x^{n}\right\| \rightarrow 0
$$

as $n \rightarrow \infty$.

Proof. Let $\varepsilon>0$; by Theorem 6 , there are integers $k, N$ and elements $a_{0}, \ldots$, $a_{N} \in A$ such that

$$
x^{k}(x-1)=\sum_{i=0}^{N} a_{i} x^{i}, \text { and } \sum_{i=0}^{N}\left\|a_{i}\right\|<\varepsilon .
$$

Choose $p>\max (k, N)$ such that, for all $n>p$,

$$
(1+\varepsilon)^{-1 / N}<\mu(n+1) / \mu(n)<(1+\varepsilon)^{1 / N} .
$$

Then, whenever $n>N+p$ and $|k-n| \leq N$, we have $\mu(k) / \mu(n) \leq 1+\varepsilon$.

But, for all $n>N+p, x^{n}(x-1)=\sum_{i=0}^{N} a_{i} x^{n-k+i}$, so that

$$
\begin{aligned}
\left\|x^{n}(x-1)\right\| & \leq \sum_{i=0}^{N}\left\|a_{i}\right\|\left\|x^{n-k+i}\right\| \leq \sum_{i=0}^{N}\left\|a_{i}\right\| \mu(n-k+i) \\
& \leq \sum_{i=0}^{N}\left\|a_{i}\right\| \mu(n)(1+\varepsilon) \leq \varepsilon(1+\varepsilon) \mu(n)
\end{aligned}
$$

and the result follows.

3. Some remarks on commutative radical Banach algebras. Let $R$ be a nonzero, commutative radical Banach algebra, and let $A=R_{+}$, the standard unitization of $R$. An element $x \in R$ will be called quasi-power-bounded (or $q p b$ ) if and only if $1+x$ is a power-bounded element of $A$. Of course, $x=0$ is always qpb; any other qpb element of $R$ will be called a non-trivial qpb element. As an immediate application of Theorem 2 :

TheOREM 7. With $R$ as above, let $x$ be a (non-trivial) qpb element of $R$. Then:

(i) $x(1+x)^{n} \rightarrow 0$ as $n \rightarrow \infty$;

(ii) let $e_{n}=1-(1+x)^{n}(n \geq 1)$; then $\left(e_{n}\right)$ is a bounded approximate identity in the closed ideal $\overline{R x}$ of $R$. 
Pr o of. (i) Since $R$ is radical, $\operatorname{Sp}_{A}(1+x)=1$. Hence, since $1+x$ is power-bounded, Theorem 2 gives that

$$
x(1+x)^{n}=(1+x)^{n+1}-(1+x)^{n} \rightarrow 0,
$$

as $n \rightarrow \infty$.

(ii) Let $e_{n}=1-(1+x)^{n}$ for $n \geq 1$. Then $\left(e_{n}\right)_{n \geq 1}$ is a bounded sequence in $R$. Moreover, for every $r \in R$,

$$
r x e_{n}=r x-r x(1+x)^{n} \rightarrow r x,
$$

as $n \rightarrow \infty$, by (i).

Since $\left(e_{n}\right)$ is bounded, it then follows that $y e_{n} \rightarrow y(n \rightarrow \infty)$ for every $y \in \overline{R x}$. But also, since again $x(1+x)^{n} \rightarrow 0$, i.e. $\left\|x+\sum_{k=1}^{n}\left(\begin{array}{l}n \\ k\end{array}\right) x^{k+1}\right\| \rightarrow 0$, then $x \in \overline{R x}$, so also $e_{n} \in \overline{R x}$, and $\left(e_{n}\right)$ is a bounded approximate identity in $\overline{R x}$.

In view of the last result, it is natural to ask whether every non-zero, commutative radical Banach algebra with a bounded approximate identity contains some non-zero qpb element. We do not know the answer to this question.

Moreover, we do not even know the answer for what is possibly the simplest example, namely the Volterra algebra $V=L^{1}[0,1]$, with the usual convolution multiplication. In connection with this algebra, let $u$ be the element $u(t)=1(0 \leq t \leq 1)$. To avoid confusion, write $\delta$ for the identity element of the unitization of $V$ (since we may identify $\delta$ with a unit point mass at 0 ). Then we have the estimates (with indices denoting convolution powers),

$$
\left\|(\delta-u)^{n}\right\|_{1}=O\left(n^{1 / 4}\right), \quad\left\|u(\delta-u)^{n}\right\|_{1}=O\left(n^{-1 / 4}\right),
$$

as $n \rightarrow \infty$. These estimates, which are the best possible estimates using just powers of $n$, are derived from classical asymptotic properties of Laguerre polynomials. We note that $u(\delta-u)^{n} \rightarrow 0$, even though $u$ is not qpb. The first of these estimates has also been noted by T. Pytlik [9].

We shall conclude by looking at some related Banach algebras. Suppose that we let $V_{c}=V_{c}[0,1]$ be the algebra of all continuous complex-valued functions on $[0,1]$, again with convolution product, and with the uniform norm $\|\cdot\|_{\infty}$. Then $V_{c}$ is a so-called uniformly radical Banach algebra, i.e. $\left\|x^{n}\right\|^{1 / n} \rightarrow 0$ uniformly on the unit sphere of $V_{c}$. It is obvious that every closed subalgebra of a uniformly radical Banach algebra is itself uniformly radical; it is a simple remark that a (non-zero) uniformly radical algebra can not contain a bounded approximate identity. Hence, by Theorem $7, V_{c}$ has no non-trivial qpb element.

Let $\|\cdot\|_{p}(p \geq 1)$ be the usual $L_{p}$-norm on $V_{c}$, and let $V_{p}$ be the completion of $V_{c}$ in this norm. Thus, as a Banach space, for $1 \leq p<\infty, V_{p}$ is the usual $L^{p}[0,1]$; it easy to see that each $V_{p}$ is a commutative radical Banach algebra for the convolution product. In fact $V_{\infty}=V_{c}$ and $V_{1}=V$, the standard Volterra algebra. It is standard that, for $1 \leq p \leq q \leq \infty$, then $V_{q} \subseteq V_{p}$ and $\|f\|_{p} \leq\|f\|_{q}$ for all $f \in V_{q}$. Evidently the same inequalities hold for the unitizations of these algebras, so that if some $V_{q}$ contained a non-trivial qpb element, the same element would be qpb in $V_{p}$ for $1 \leq p \leq q$. We have just seen that $V_{\infty}=V_{c}$ has no non-trivial qpb element. We shall now show that this holds for every $p>1$. 
THEOREM 8. For every $1<p \leq \infty, V_{p}$ has no non-trivial qpb element.

Pr o of. We just have to consider the case $1<p<\infty$. But then $V_{p}$ is a dual space, and multiplication in $V_{p}$ is separately continuous for the weak-* topology. Suppose that $x$ is a qpb element of $V_{p}$. Then the sequence $e_{n}=\delta-(\delta+x)^{n}$, which by Theorem 7 is a bounded approximate identity for $\overline{V_{p} x}$, has a weak-* cluster point, say $y$. Using the separate weak-* continuity of multiplication, it follows that $x y=x$. Since $V_{p}$ is a radical algebra, $\delta-y$ is invertible in the unitization of $V_{p}$ and it follows that $x=0$.

There is, however, one other good norm to take on $V_{c}$ - or even on $V_{1}$. We define $\|\cdot\|_{o p}$ to be the norm as a convolution operator on the Hilbert space $H=L^{2}[0,1]$. Thus, explicitly, for $f \in V_{1}$ we define

$$
\|f\|_{o p}=\sup \left\{\|f * g\|_{2}: g \in V_{2},\|g\|_{2} \leq 1\right\} .
$$

It is standard that $\|f\|_{o p} \leq\|f\|_{1}$ for every $f \in V_{1}$. We let $V_{o p}$ be the completion of $V_{1}$ in $\|\cdot\|_{o p}$. It is clear that $V_{o p}$ may also be described as the norm-closed subalgebra of $L(H)$ generated by the Volterra integration operator $U$, where

$$
U(f)(x)=(u * f)(x)=\int_{0}^{x} f(t) d t,
$$

and here $u$ is, as before, the function $u(t) \equiv 1$. We shall see that the element $-u$ is a qpb element of $V_{o p}$, whereas, as we saw above, for the larger $\|\cdot\|_{1}$, we merely had $\left\|(\delta-u)^{n}\right\|_{1}=O\left(n^{1 / 4}\right)$.

THEOREM 9. The function $-u$ is $q p b$ in $V_{o p}$.

Proof. We have to show that, with the above notation, $I-U$ is a power-bounded element of $L(H)$.

It is a familiar fact (see e.g. Halmos [6], Problem 150) that $\left\|(I+U)^{-1}\right\|=1$, so that $(I+U)^{-1}$ is certainly power-bounded. (In fact this already shows that $\delta-(\delta+u)^{-1}$ is a non-trivial qpb element of $V_{o p}$.)

The link with $I-U$ comes from an ingenious remark (made to the author by T. V. Pedersen) that $I-U$ and $(I+U)^{-1}$ are similar, as elements of $L(H)$.

In fact, recalling that $H=L^{2}[0,1]$, we define $S \in L(H)$ by

$$
(S f)(t)=e^{t} f(t) \quad(f \in H) .
$$

Then

$$
\begin{aligned}
\left(S^{-1} U S\right) f(x) & =e^{-x} \int_{0}^{x} e^{t} f(t) d t=\int_{0}^{x} e^{-(x-t)} f(t) d t \\
& =\sum_{n=0}^{\infty} \int_{0}^{x} \frac{(-1)^{n}(x-t)^{n}}{n !} f(t) d t=\sum_{n=1}^{\infty}(-1)^{n-1}\left(u^{n} * f\right)(x) \\
& =f(x)-(I+U)^{-1} f(x),
\end{aligned}
$$

i.e. $(I+U)^{-1}=S^{-1}(I-U) S$, which completes the proof. 


\section{References}

[1] G. R. Allan, A. G. O'Farrell and T. J. Ransford, A tauberian theorem arising in operator theory, Bull. London Math. Soc. 19 (1987), 537-545.

[2] G. R. Allan and T. J. Ransford, Power-dominated elements in a Banach algebra, Studia Math. 94 (1989), 63-79.

[3] R. P. Boas, Entire Functions, Academic Press, New York, 1954.

[4] J. Esterle, Quasimultipliers, representations of $H^{\infty}$, and the closed ideal problem for commutative Banach algebras, in: Radical Banach Algebras and Automatic Continuity, Proc. Conf. Long Beach 1981, J. Bachar et al. (eds.), Lecture Notes in Math. 975, Springer, Berlin, 1983, 66-162.

[5] I. Gelfand, Zur Theorie der Charaktere der abelschen topologischen Gruppen, Rec. Math. N.S. (Mat. Sb.) 9 (51) (1941), 49-50.

[6] P. R. Halmos, A Hilbert Space Problem Book, Van Nostrand, Princeton 1967.

[7] E. Hille and R. S. Phillips, Functional Analysis and Semi-groups, Amer. Math. Soc. Colloq. Publ. 31, Providence, R.I., 1957.

[8] Y. Katznelson and L. Tzafriri, On power-bounded operators, J. Funct. Anal. 68 (1986), $313-328$.

[9] T. Pytlik, Analytic semigroups in Banach algebras, Colloq. Math. 51 (1987), 287-294. 